\title{
PREDICTIVE DIAGNOSIS OF FATAL HEART RHYTHMS USING WEARABLES
}

\author{
Jeno Szep \\ Salim Hariri \\ Department of Electrical and Computer Engineering \\ The University of Arizona \\ 1230 E. Speedway Boulevard \\ Tucson, AZ, USA \\ \{szep, hariri\}@email.arizona.edu
}

\author{
Zain Khalpey
}

\author{
Department of Surgery \\ The University of Arizona \\ 1501 N. Campbell Avenue \\ Tucson, AZ, USA \\ zkhalpey@surgery.arizona.edu
}

\begin{abstract}
Sudden cardiac death causes more than 300,000 deaths annually in the US. Our research goal is to develop a continuous cardiac monitoring system that utilizes current wearable devices and is capable of not just detecting arrhythmia but also to predict life-threatening arrhythmia a few minutes before it would actually happen. The monitoring system should provide a diagnosis based on analyzing a few-minutes of heart-rate data streams. In order to verify the feasibility of this approach, we have developed a prototype and evaluated its capabilities. The prototype is based on a two-tier data analytics approach and utilizes multiple gradient boosting machine learning models. The system was tested for predicting four different life-threatening arrhythmias solely on realistic heart-rate readings and also tested the atrial fibrillation recognition capability. The prototype scored $91.6 \%$ and $93.9 \%$ accuracy respectively. These preliminary results validate the feasibility of our approach to predict arrhythmia in real-time from heart-rate observations.
\end{abstract}

Keywords: Prediction of Arrhythmia, Heart Monitoring, Machine Learning, Smartwatch.

\section{INTRODUCTION}

Sudden Cardiac Arrest (SCA) is a leading cause of death in the United States, claiming an estimated 325,000 lives each year (SCAF 2019). Outside hospitals SCA occurs most often in a home or residence. Time is critical because the chances of survival from SCA decrease by $10 \%$ every minute. There is a need for an easy-to-use affordable solution that provides continuous monitoring of the heart rhythm, detects arrhythmia and also provides a prediction of possible life-threatening heart rhythms (LTHR) at least a few minutes prior to such an event. While such a technology would be beneficial for everyone, certain segments of the population are in special need because of higher risk of SCA. Such cohorts are elderly people, athletes, military, and cardiovascular disease patients.

New wearable technology, like smartwatches, provide affordable continuous heart-rate (HR) monitoring and communication possibilities, while cloud-based services can add data storage and effective analytics. Current research interest in the area focuses primarily on the automated identification of different arrhythmias using electrical sensors (ECG) or photoplethysmography sensors (PPG).

Significant research has been done in the area of using clinical physiological data for automatic detection of heart arrhythmia. Most of the research is focusing on the detection of atrial fibrillation (AF) because AF is the most common type of arrhythmia. Arrhythmia can be detected from the features - like the length of inter-beat intervals - obtained from electrical sensors (ECG) (Huang et al. 2011, Rincon 2012, Datta et al. 
2017, Hong et al. 2017, Zabihi et al. 2017) and optical photoplethysmography sensors (PPG) (Tang et al. 2017, Krivoshei et al. 2017, Bonomi et al. 2018, Stanford Medicine 2018). The inter-beat interval-based data analytics uses a variety of heart-rate variation (HRV) related features. Shaffer and Ginsberg (2017) provide a good overview of the most common HRV features. A subcategory of the HRV features is related to the Poincare-plot, where the lengths of inter-beat intervals are plotted on a graph as a function of the lengths of the previous one. Some researchers detect tachycardia or AF solely from the shape of the Poincare plot (Sarkar et al. 2008, Park et al. 2009, Tuboly and Kozmann 2015). It is more common however, that the shape of the Poincare plot is quantified and the obtained features are used together with other statistical HRV features in the classification process (Esperer and Oehler 2010). Classification algorithms stretch to a wide spectrum, including clustering (Park et al. 2009), nonparametric statistical tests (Huang et al. 2011), fuzzy classification (Rincon 2012), logistic regression (Tang et al. 2017), Markov models (Bonomi et al. 2018), random forest (Zabihi et al. 2017), two-layer binary classifier (Datta et al. 2017), DNN (Ballinger et al. 2018), LSTM networks (Teijeiro et al. 2017) and gradient boosting (Hong et al. 2017, Teijeiro et al. 2017).

Regarding the prediction of LTHR, it was shown that the dynamism of heart rate changes before AF (Vikman et al. 1999), there are ectopic beats two minutes before AF (Hnatkova et al. 1998), and spectral analysis of the HR shows changes preceding AF (Herweg et al. 1998). Lee et al. (2016) predicted VT one hour before onset based on the ECG-signal and respiration rate with $85.3 \%$ accuracy. There is however no working model on real-time LTHR prediction from realistic HR data.

Research also shows the versatility of continuous monitoring: Ballinger (2018) used one-week long HRV and step-count monitoring data from wearable sensors to recognize multiple medical conditions including diabetes, high cholesterol, high blood pressure, and sleep apnea, indicating that in the future continuous monitoring can yield additional health benefits besides cardiac monitoring.

In our approach, we envision a complex system, the Continuous Heart Monitoring, Analysis, Prediction and Diagnosis (CHMAPD) system that will provide continuous monitoring and personalized diagnosis, utilizing the heart-rate sensing capability of widely available common smartwatches. The CHMAPD system will predict LTHR and detect multiple types of irregular heart rhythms like atrial fibrillation (AF), premature ventricular contractions (PVC) in real time. The envisioned system is described in more details in the section on Future Work.

To investigate the feasibility of the approach we designed a pilot system that uses heart-rate values and recognize LTHR a few minutes before the onset of the event, and can identify the most common arrhythmia (atrial fibrillation). The rest of the paper is focused on this pilot system and the results obtained.

Smartwatches detect blood volume changes with a photoplethysmography sensor, process the raw sensory data in the spectral domain and filter the result for feasibility (Garde 2014, Orphanidou 2018). This proprietary process results in smoothed heart-rate values calculated in every 1.5-3 seconds unless motion artifacts disrupt the process. The HR values calculated this way have an average $2-5 \%$ error rate (Shcherbina 2017). These HR values are available through an API for our prototype. The fact that consecutive HR readings of a smartwatch are not related to consecutive inter-beat intervals, does not allow for accurate calculation of HRV and Poincare plot related features in our model. The above listed related research works have used either the ECG data or the raw data from PPG sensors, that enabled the calculation of relatively accurate consecutive inter-beat intervals. Therefore, a major challenge for our prototype is to detect AF with good accuracy by using the HR readings of smartwatches that have limited accuracy.

In our approach, the prediction/recognition algorithm of LTHR is based on a two-tier machine learning algorithm with multiple regressive ML models. The two-tier approach increases prediction accuracy. The input is the continuous heart-rate (HR) data stream provided by a wearable sensor like a smartwatch. In Tier-1 the HR data is divided into one-minute long overlapping packets. Heart-rate variability (HRV) features from the time domain, information theory domain, frequency domain, and non-linear domain are calculated for each packet. Three different gradient boosting regression models are used in Tier-1. In Tier- 
2 larger units, 'blocks' are formed. 4-10 packets are grouped into single blocks and are tagged with features that are the predicted values from Tier-1. In Tier-2 another gradient boosting classifier model is applied. The performance of the pilot system was tested with realistic noisy data in two different scenarios: predicting LTHR several minutes before onset, and the recognition of AF.

The rest of this paper is organized as follows. Section 2 details our methods used in the prototype, Section 3 provides an overview of the results, and finally, Section 4 summarizes the paper and provides an outline of our future research plans.

\section{METHODS}

The pilot system utilizes a two-tier machine learning approach for effective detection in changes in HR patterns for the prediction of life-threatening heart rhythm and recognition of atrial fibrillation. Briefly, in Tier-1, the incoming HR data stream is divided into short-range packets and we then determine the HRV features associated with these packets. In Tier-2, blocks of packets are created representing 2-4 minutes of the data stream. The algorithm is explained in details below.

The scheme of the two-tier machine learning training pipeline is shown in Figure 1. Practically the same design can be used both for training the models and also for the continuous operation of the system. During the training phase, the appropriate features are selected and the model is tuned to enhance the performance. During the operation phase, the input data stream feeds the trained models in order to perform the prediction.

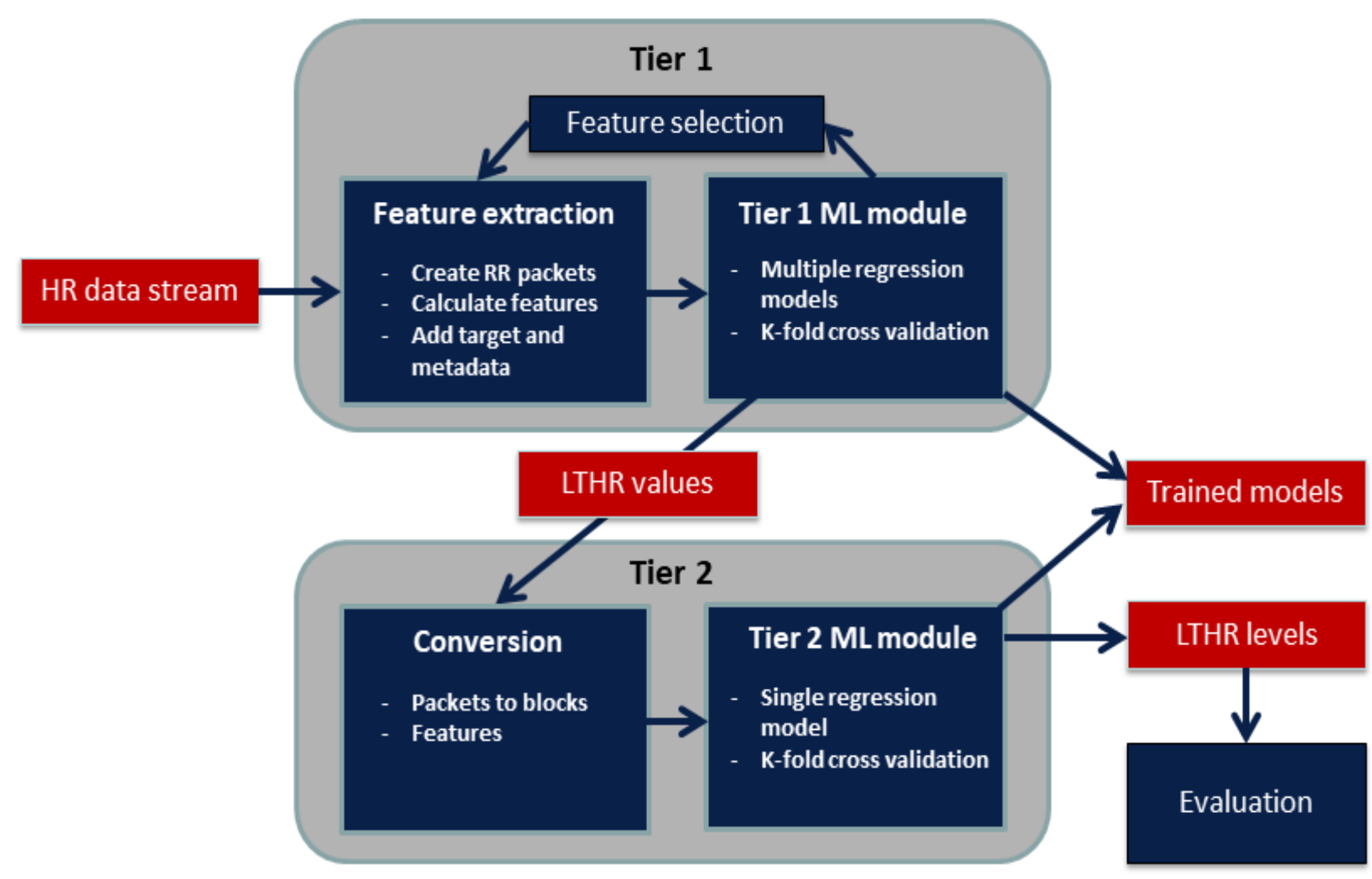

Figure 1: Machine learning training pipeline in the two-tier approach. 


\subsection{Tier-1}

The incoming HR data is divided into 60 seconds long overlapping packets with $15 \mathrm{~s}$ shifts, and for each packet, the HRV features are calculated. No packet is formed with less than 25 HR values. The HR values are converted to virtual heartbeat intervals (RR) and also the differences of consecutive RR values are calculated (DRR). It is worth mentioning that due to the process of how a smartwatch calculates the HR values, the DRR values are not equal to the differences of consecutive inter-beat intervals. Table 1 summarizes the HRV features we have used.

Table 1: HRV features used in the model.

\begin{tabular}{|l|l|}
\hline $\begin{array}{l}\text { Number of } \\
\text { Features }\end{array}$ & Description \\
\hline \multicolumn{2}{|c|}{ Time Domain/Statistical Features } \\
\hline 22 & $\begin{array}{l}\text { Different statistical properties of RR and DRR values, like mean, standard } \\
\text { deviation, skewness, etc. }\end{array}$ \\
\hline 8 & \multicolumn{1}{c|}{ Information Theory Domain Features } \\
\hline \multicolumn{2}{|c|}{$\begin{array}{l}\text { Different entropy related features of RR and DRR, like Shannon entropy and } \\
\text { Rényi entropy }\end{array}$} \\
\hline 6 & $\begin{array}{l}\text { Features obtained from the fast Fourier transform, describing the power in } \\
\text { different frequency bands and their relations }\end{array}$ \\
\hline & \multicolumn{2}{c|}{$\begin{array}{l}\text { Features describing the statistics of the points on the Poincaré plot of RR and } \\
\text { DRR values, like standard deviation along the line of identity and standard } \\
\text { deviation perpendicular to the line of identity, etc. }\end{array}$} \\
\hline 12 & \multicolumn{2}{c}{} \\
\hline
\end{tabular}

The target function for training is binary when the pilot system is used for AF detection, however, it is a linearly increasing function with time when the system is used for predicting LTHR. In this latter case packet $i$ is tagged with a target value $T_{i}=\operatorname{ReLU}\left(0.63-\left(t_{0}-t_{i}\right) / 700\right)$ where $t_{i}$ is the mean of $\mathrm{HR}$ timestamps in packet $i, t_{0}$ denotes the time of LTHR onset, and ReLU is the rectifier linear unit function (time is measured in s). In the prototype, we are using multiple parallel tree boosting machine learning models (boosted tree models) that can solve many data science problems in a fast and accurate way. The three gradient boosting models in Tier-1 are: the XGB Regressor (XGBoost 2016), the Light GBM Regressor (LightGBM 2017), and CatBoost Regressor (CatBoost 2018). A 5-fold cross-validation is used with ensuring that no overlapping packets are in different folds.

The prediction values for each packet predicted by the trained models (LTHR values) are passed to Tier-2 as input values.

\subsection{Tier-2}

In Tier-2 the blocks are formed from a given number $(\mathrm{P}=4,6,8,10)$ of packets. In the case of LTHR prediction, one block was formed only for each sample because of the limited length of the data samples. At AF detection multiple overlapping blocks were formed. The block features are then the prediction values calculated in Tier-1 for each packet by each model, therefore the number of features in Tier-2 is $3 \mathrm{xP}$ where $\mathrm{P}$ denotes the number of pockets per block. This method can be considered as an extended version of the classical stacking technique because it stacks not only the different models, but it extends in the time dimension also. 
The training target function in Tier-2 is always binary for both AF detection and also for LTHR prediction.

Single gradient boosting classifier model is applied (XGB Regressor). 5-fold cross-validation is used with ensuring that overlapping blocks are always in the same fold. The prediction values for each block are calculated by the trained Tier-2 model, and these values are the final detected value of pre-LTHR or AF level by the pilot system.

\subsection{Test Data}

LTHR Prediction: The data were obtained from the PhysioNet 2015 challenge (PhysioNet 2015). The data was recorded by ICU monitors leading up to a total of 750 life-threatening arrhythmia alarms recorded from three of the largest intensive care monitor manufacturers' bedside units. LTHR included asystole, extreme bradycardia, extreme tachycardia, ventricular tachycardia, and ventricular flutter/fibrillation. The record length varies between 6-6.5 minutes and in all 750 cases an event at time 4:50-5:00 triggered the built-in alarm at the ICU. In almost half of the cases, the alarm was false, these cases are marked. From the four data channels provided, we have used only the data in the PPG channel and we also kept the first 4 minutes of the record only. Most of the data files had significant noise artifacts (likely due to motion). We kept those 486 files where the HR detection was not interrupted for more than one minute. $51 \%$ of the selected files contained true LTHR. The heart rate was obtained from the raw PPG signal by a custom written software based on wavelet transformation. There was a minimum of 1.5 seconds between two successive heart-rate values in order to simulate more realistically a smart-watch data stream.

AF detection: Heart rate values recorded by the PPG sensor of a Polar watch was recorded in a clinical environment from 12 persons. Data included 4.8 hours of AF, and 5.9 hours of normal sinus rhythm (NSR). Continuous data recordings varied between 8 minutes to 42 minutes. The Polar watch provides an HR read in approximately every one and a half seconds, however, due to artifacts, the average time between two consecutive reads was 4.3 seconds. All participants provided written informed consent.

Both datasets contained realistic noisy heart-rate recordings with artifacts affecting more than 10 percent of the data.

\section{RESULTS}

Tests were performed with a different number of packets per blocks. As expected, the results were more accurate when more packets formed the blocks, meaning that prediction based on a longer observation period is more accurate. With $\mathrm{P}=4$ packets per blocks, the length of the data stream in a block was about 2 minutes, while with $\mathrm{P}=10$ packets per block it was about 3.5-4 minutes.

LTHR Prediction: The area under the ROC curve (Haijan-Tilaki 2013) for different P values is shown in Figure 2. The dataset contained HR values from about 5 minutes before the onset of LTHR to 1 minute before it. The $\mathrm{P}=4$ predictions are based on data from 5 minutes to 3 minutes before LTHR onset, while the $\mathrm{P}=10$ predictions are based on data from 5 minutes to 1 minute before onset. LTHR included Asystole, Bradycardia, Ventricular Flutter Fibrillation, Tachycardia, Ventricular Tachycardia.

The $\mathrm{P}=10$ version was able to distinguish the pre-LTHR rhythms from non-pre-LTHR ones with ROCAUC of 0.966 and accuracy (true positives plus true negatives per all) of $91.6 \%$. Figure 3 a shows the ROCAUC curve, and Figure $3 b$ shows the accuracy as a function of the threshold value for the $\mathrm{P}=10$ cases. 


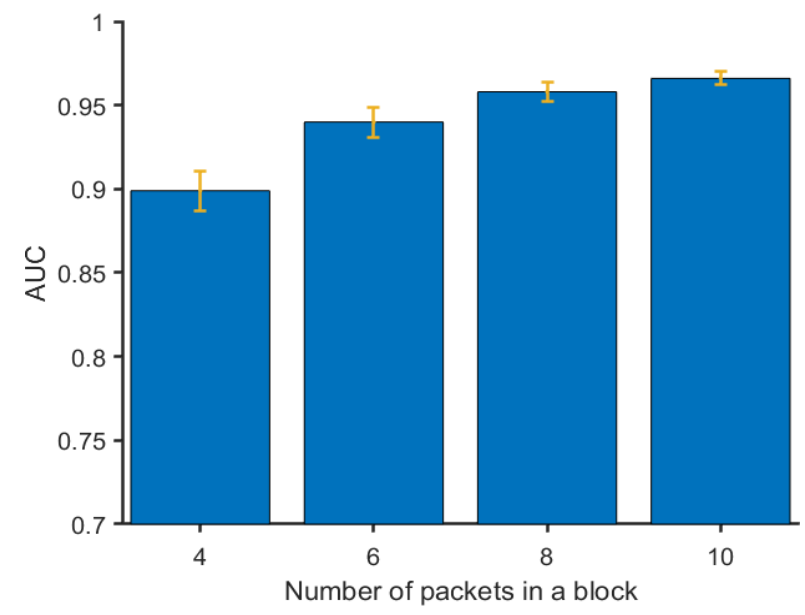

Figure 2: Area under the ROC curve for different number of packets in a block. The error bars represent the standard error of the mean, based on five trainings with different randomization in in the cross validation.
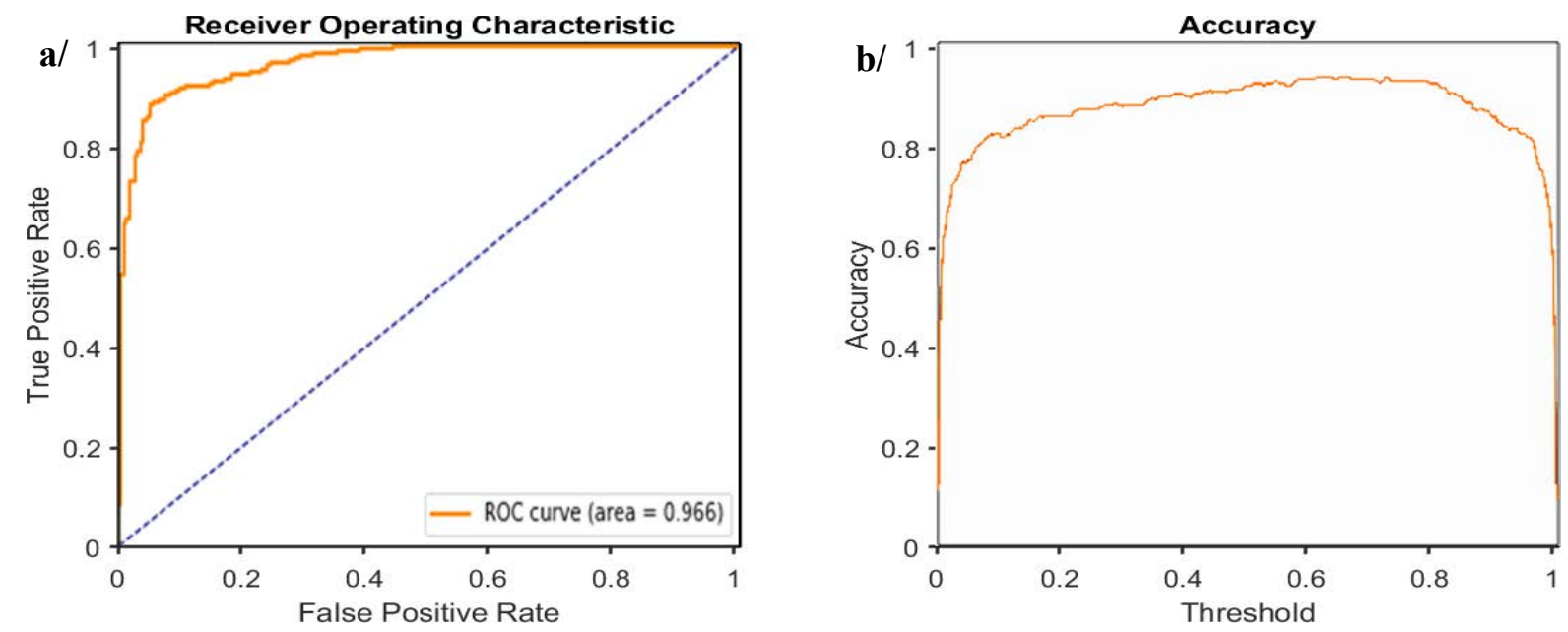

Figure 3: Results of LTHR prediction for the $\mathrm{P}=10$ case: $\mathbf{a} /$ The ROC curve. $\mathbf{b} /$ The accuracy (true positives plus true negatives per all) as function of the threshold, with a maximum value of $91.6 \%$.

AF detection: The test results for AF detection based on 3.5-4 minutes long samples $(\mathrm{P}=10)$ were ROCAUC of 0.968 and accuracy of $93.9 \%$ as shown in Figure 4. Although as we mentioned earlier our input HR data had limited accuracy, our results are comparable to the accuracy of the AF detection algorithms $(92-99 \%)$ listed above in the Introduction. 

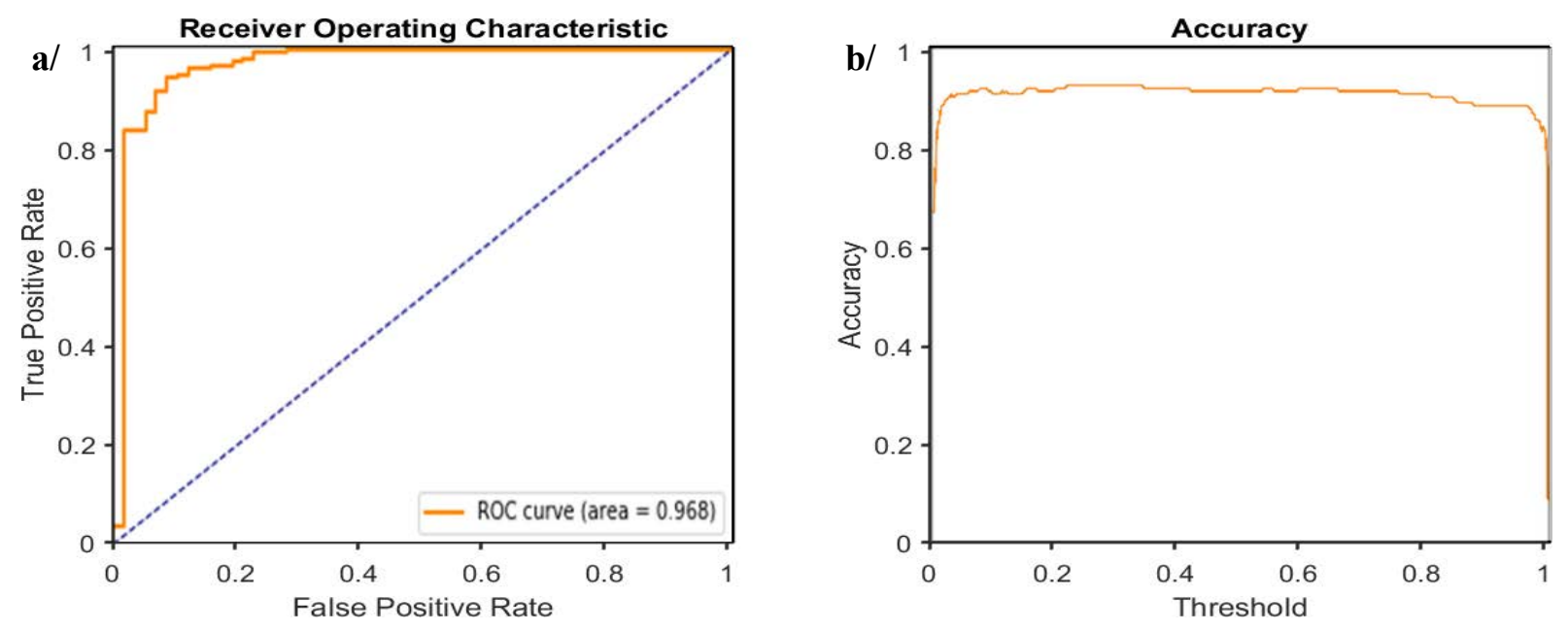

Figure 4: Results of AF recognition for the $\mathrm{P}=10$ case. $\mathbf{a} /$ The ROC curve. $\mathbf{b} /$ The accuracy as function of the threshold, with a maximum value of $93.9 \%$.

\section{SUMMARY AND FUTURE WORK}

In this paper, we presented a prototype cardiac monitoring system that utilizes noisy realistic heart-rate readings obtained partially by smartwatches and is capable of not just detecting arrhythmia but also to predict life-threatening arrhythmia a few minutes before it actually happens. Our preliminary results showed that our prototype scored $91.6 \%$ accuracy (AUC 0.966) on predicting LTHR, which is a novelty. We also tested the atrial fibrillation recognition capability. By training the model on heart-rate recordings of 10.7 hours total, we obtained $93.9 \%$ accuracy (AUC 0.968 ). These preliminary results on AF-detection, based only on realistic heart-rate readings are comparable to the state-of-the-art AF detection algorithms that are based on more accurate clinical data. These results validate the feasibility of our approach to predict/detect accurately arrhythmia in real-time.

Our future work in the short-term includes testing the scheme's accuracy with recurrent neural networks, specifically with Long Short-Term Memory (LSTM) networks, furthermore testing the effectivity of a live prototype in a real-life environment. A bottleneck in the current prototype is that we need to retrain the boosted-tree models whenever new training data becomes available, while the use of LSTM networks would inherently solve the life-long learning requirement in the future.

Our long-term research goal is to develop a complex physiological monitoring system, the Continuous Heart Monitoring, Analysis, Prediction and Diagnosis (CHMAPD) system that

- Provides continuous monitoring

- Easily accessible for a large user base, utilizing widely available smartwatches

- Ensures high availability

- Has an analytical capability that can be customized to each user

- Predicts and detects life-threatening arrhythmia

- Communicates the detected status with the user, medical care and ambulance

The architecture of the proposed Continuous Heart Monitoring, Analysis, Prediction, and Diagnosis system is shown in Figure 5. The source of the Cardiac Data Stream is the heart-rate data sensed by the PPG sensor of a smartwatch and transferred to the cloud-based Data-Driven Personalized Cardiac Analytics system by a smartphone app. A critical part of the proposed system is the capability of predicting LTHR and detecting 
arrhythmia. This capability should be functional in a real-life environment, with noisy data, and provide results on the basis of a few minute-long data stream.

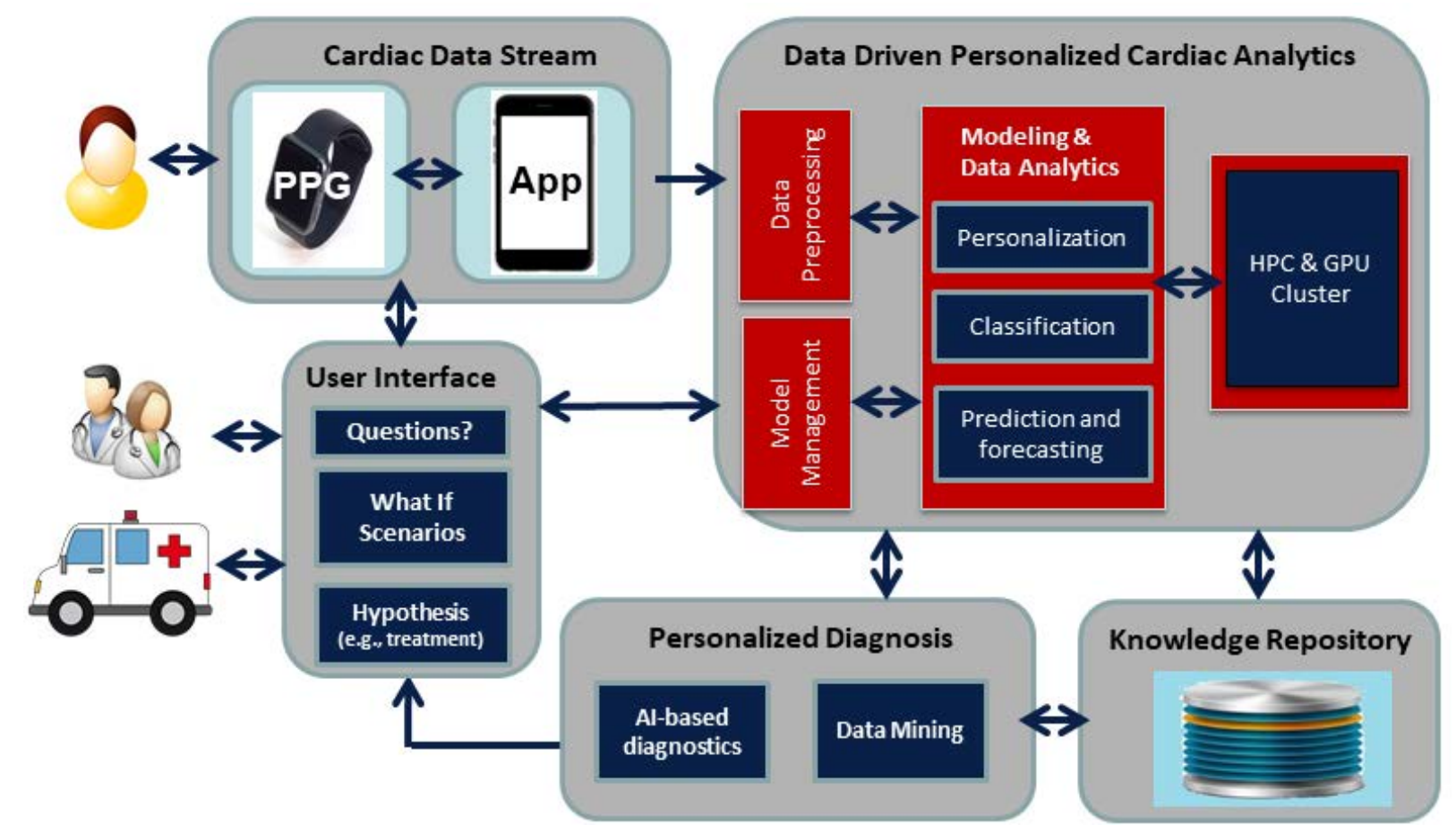

Figure 5: The architecture of the Continuous Heart Monitoring, Analysis, Prediction and Diagnosis system.

The proposed system will be accessable for users by downloading an app from the App Store, while the Personalized Cardiac Analytics and Diagnosis will run in the AWS cloud.

\section{ACKNOWLEDGMENTS}

This work is partly supported by the Air Force Office of Scientific Research (AFOSR) Dynamic DataDriven Application Systems (DDDAS) award number FA9550-18-1-0427, National Science Foundation (NSF) research projects NSF-1624668 and SES-1314631, and the All Heart Foundation.

\section{REFERENCES}

Ballinger, B., J. Hsieh, A. Singh, N. Sohoni, ..., M. J. Pletcher. 2018. "DeepHeart: Semi-Supervised Sequence Learning for Cardiovascular Risk Prediction”. The Thirty-Second AAAI Conference on Artificial Intelligence (AAAI-18), pp. 2079-2086

Bonomi, A. G., F. Schipper, L. M. Eerikainen, ..., L. R. C. Dekker. 2018. “Atrial Fibrillation Detection Using a Novel Cardiac Ambulatory Monitor Based on Photo-Plethysmography at the Wrist". Journal of American Heart Association, vol. 7:e009351. DOI: 10.1161/JAHA.118.009351

CatBoost 2018. Developed by Yandex, https://github.com/catboost. Accessed on Feb. 17. 2019

Datta, S., C. Puri, A. Mukherjee, R. Banerjee, ..., S. Khandelwal 2017. "Identifying Normal, AF and other Abnormal ECG Rhythms using a Cascaded Binary Classifier". Computing in Cardiology, vol 44, pp. 1-4., DOI: 10.22489/CinC.2017.173-154 
Esperer, H. D. and M. Oehler. 2010. "Automatic quantification of the Poincare plot asymmetry of NNinterval recordings". Physiological Measurement, vol. 31, pp. 395-413, DOI: 10.1088/0967$3334 / 31 / 3 / 008$

Garde, H., W. Karlen, J. M. Ansermino, G. A. Dumont. 2018. "Estimating Respiratory and Heart Rates from the Correntropy Spectral Density of the Photoplethysmogram". PLOS ONE, vol. 9(1).

Herweg, B., P. Dalal, B. Nagy, and P. Schweitzer. 1998. "Power Spectral Analysis of Heart Period Variability of Preceding Sinus Rhythm Before Initiation of Paroxysmal Atrial Fibrillation". American Journal of Cardiology, vol. 82, pp. 869-874

Haijan-Tilaki, K. 2013. "Receiver Operating Characteristic (ROC) Curve Analysis for Medical Diagnostic Test Evaluation". Caspian J Intern Med, vol.4(2), pp. 627-635

Hnatkova, K., J. E. P. Waktare, F. D. Murgatroyd, ..., M. Malik. 1998. "Analysis of the cardiac rhythm preceding episodes of paroxysmal atrial fibrillation". American Heart Journal, vol. 135, pp. 1010-1019

Hong, S., M. Wu, Y. Zhou, ..., J. Xie. 2017. "ENCASE: an ENsemble ClASsifiEr for ECG Classification Using Expert Features and Deep Neural Networks". Computing in Cardiology, vol. 44 pp. 1-4, DOI: 10.22489/CinC.2017.178-245

Huang, C., S. Ye, H. Chen, D. Li, F. He, and Y.Tu. 2011. "A Novel Method for Detection of the Transition Between Atrial Fibrillation and Sinus Rhythm". IEEE Transactions on Biomedical Engineering, vol. 58, NO. 4, pp. 1113-1119

Krivoshei, L., S. Weber, T. Burkard, ..., J. Eckstein1. 2017. "Smart detection of atrial fibrillation". Europace, vol. 19, pp. 753-757. DOI:10.1093/europace/euw125

Lee, H., Shin, S-Y., Seo M., Nam, G-B. and Joo, S. 2016. "Prediction of Ventricular Tachycardia One Hour before Occurrence Using Artificial Neural Networks". Scientific Reports, vol. 6:32390, DOI: 10.1038

LightGBM, 2017. Light Gradient Boosting Framework by IBM, https://lightgbm.readthedocs.io/en/latest/genindex.html. Accessed on Feb. 17. 2019

Lim, W. K., S. Davila, J. X. Teo, ..., P. Tan. 2018. "Beyond fitness tracking: The use of consumer-grade wearable data from normal volunteers in cardiovascular and lipidomics research". PLoS Biol vol. 16(2): e2004285. DOI: 10.1371/journal.pbio.2004285

Orphanidou, C. 2018. Signal Quality Assessment in Physiological Monitoring, State of the Art and Practical Considerations. SpringerBriefs in Bioengineering, ISBN 978-3-319-68414-7, DOI: 10.1007/978-3319-68415-4

Park, J., Lee, S. and Jeon, M. 2009. "Atrial fibrillation detection by heart rate variability in Poincare plot". BioMedical Engineering OnLine, vol. 8:38 DOI: 10.1186/1475-925X-8-38

PhysioNet 2015. Computing in Cardiology Challenge, https://physionet.org/challenge/2015/\#challengedata. Accessed on Jan. 04. 2019

Rincon, F., R. P. Grassi, N. Khaled, D. Atienza, and D. Sciuto, 2012. "Automated Real-Time Atrial Fibrillation Detection on a Wearable Wireless Sensor Platform". Proceedings of the 34th Annual International Conference of the IEEE EMBS, San Diego, California USA, 28 August - 1 September 2012 pp. 2472-2475.

Sarkar, S., D. Ritscher, and R. Mehra. 2008. "A Detector for a Chronic Implantable Atrial Tachyarrhythmia Monitor". IEEE Transactions on Biomedical Engineering, vol. 55.3, pp. 1219-1224. DOI: 10.1109/TBME.2007.905487

SCAF 2019. Sudden Cardiac Arrest Foundation, http://www.sca-aware.org/about-sca. Accessed on 01-042019.

Shcherbina, A., Mattsson, C M., Waggott, D., Salisbury, H., Christle, J W. et al. 2017. "Accuracy in WristWorn, Sensor-Based Measurements of Heart Rate and Energy Expenditure in a Diverse Cohort". Journal of personalized medicine, vol. 7(2): E3, DOI: 10.3390/jpm7020003 
Shaffer, F. and J. P. Ginsberg 2017. "An Overview of Heart Rate variability Metrics and Norms". Frontiers in Public Health, vol. 5:258, pp. 1-17, DOI: 10.3389/fpubh.2017.00258

Stanford Medicine 2018 Apple Heart Study, https://med.stanford.edu/appleheartstudy.html. Accessed Jan.4. 2019

Tang, S. C., Huang, P. W., Hung, C. S., ..., Jeng, J. S. 2017. "Identification of Atrial Fibrillation by Quantitative Analyses of Fingertip Photoplethysmogram". Scientific Reports, vol. 7:45644, DOI: $10.1038 /$ srep45644

Teijeiro, T., García, C. A., Castro, D. and Félix, P. 2017. "Arrhythmia Classification from the Abductive Interpretation of Short Single-Lead ECG Records", Computing in Cardiology, vol. 44, pp. 1-4., DOI:10.22489/CinC.2017.166-054

Tuboly, G., Kozmann, G. 2015. “Atrial Fibrillation Detection Based on Poincaré plot of RR Intervals". MEASUREMENT, Proceedings of the 10th International Conference, Smolenice, Slovakia, pp. 89-92

Vikman, S., Makikallio, T. H., Yli-Mayry, S., ..., Huikuri, H. V. 1999. "Altered Complexity and Correlation Properties of R-R Interval Dynamics Before the Spontaneous Onset of Paroxysmal Atrial Fibrillation". Circulation, vol. 100, pp. 2079-2084.

XGBoost, 2016. Extreme Gradient Boosting Framework by the Distributed Machine Learning Community (DMLC) group., https://xgboost.readthedocs.io/en/latest/. Accessed on Feb. 17. 2019

Zabihi M., A. B. Rad, A. K. Katsaggelos, ..., M. Gabbouj. 2017. "Detection of Atrial Fibrillation in ECG Hand-held Devices Using a Random Forest Classifier". Computing in Cardiology, vol 44, DOI: 10.22489/CinC.2017.069-336

\section{AUTHOR BIOGRAPHIES}

JENO I SZEP is a Research Scientist at the Department of Electrical and Computer Engineering at The University of Arizona. He received an M.S. in Mathematics and a Ph.D. degree in Physics at the Eotvos University Budapest. Earlier he was an associate professor at the Department of Solid-State Physics at the Eotvos University. His current research interest includes dynamical systems, machine learning, data analytics, modeling, and simulation. His email address is szep@email.arizona.edu.

ZAIN KHALPEY is an Associate Professor at the Department of Surgery at the University of Arizona. His email address is zkhalpey@surgery.arizona.edu.

SALIM HARIRI is a Professor in the Department of Electrical and Computer Engineering at The University of Arizona. He received his Ph.D. in computer engineering from the University of Southern California in 1986, MSc from The Ohio State University in 1982 and B. S. from Damascus University in 1977. He is the UA site director of NSF Center for Cloud and Autonomic Computing and he is the EditorIn-Chief for the CLUSTER COMPUTING JOURNAL (Springer, http://clus.edmgr.com). He is the Founder of the IEEE/ACM International Symposium on High-Performance Distributed Computing (HPDC) and the co-founder of the IEEE International Conference on Cloud and Autonomic Computing. $\mathrm{He}$ is co-author/editor of four books on Autonomic computing, parallel and distributed computing: "Autonomic Computing: Concepts, Infrastructure, and Applications" (CRC Press, 2007), "Tools and Environments for Parallel and Distributed Computing" (Wiley, 2004), and "Virtual Computing: Concept, Design and Evaluation" (Kluwer, 2001), and "Active Middleware Services" (Kluwer, 2000). Research interests include Cybersecurity modeling and analysis, resilient cyber cloud services, and high-performance parallel and distributed systems. His email address hariri@ece.arizona.edu. 